\title{
Folates and prevention of neural-tube diseases
}

\author{
Leanza V., Stracquadanio M., Ciotta L., Pafumi C., Giannone T. T.,Giunta M. R., Leanza G., \\ Mayada Chammas", Fawzi Chammas", Zarbo G.
}

Istituto Di Patologia Ostetrica e Ginecologica, Azienda Ospedaliera Universitaria Policlinico - Vittorio Emanuele, Catania - Direttore Prof. Giuseppe Zarbo

\author{
Email address: \\ pafumi@unict.it (M. Chammas)
}

\section{To cite this article:}

Leanza V., Stracquadanio M., Ciotta L., Pafumi C., Giannone T. T., Giunta M. R., Leanza G., Mayada Chammas, Fawzi Chammas*, Zarbo G..Folates and Prevention of Neural-Tube Diseases, Science Journal of Clinical Medicine. Vol. 2, No. 2, 2013 , pp. 47-51. doi: $10.11648 /$ j.sjcm.20130202.13

\begin{abstract}
Neural tube defects (NTDs) are congenital malformations of the brain and spinal cord caused by failure of the neural tube to close between 21 and 28 days following conception. Each year almost 400.000 infants are born with NTDs all over the world. Although many advances have been made in the spina bifida's treatment and have led to an increased life expectancy and an improved life quality, no treatment exists that will completely eliminate the serious disability or premature mortality associated with it. For such a reason, reducing the risk of NTDs is an important goal. It is widely accepted that adequate maternal consumption of folic acid before pregnancy and during the early weeks of gestation can reduce the danger of having a child with a neural tube defect (NTD). As a result, public health authorities worldwide have recommended consuming $400 \mu \mathrm{g}$ folic acid per day during the periconceptional period in order to decrease the risk of first occurrence NTDs. This recommended dose is based mainly on the amount of folic acid associated with a reduction in NTDs in the majority of epidemiological studies. Literature data asserts that folic acid can prevent about $70 \%$ of NTD conceptions while folic acid-containing multivitamin can prevent about $90 \%$; the diagnostic efficacy of ultrasound is nearly $100 \%$ in anencephalic fetuses and about $80 \%$ in fetuses with different manifestations of spina bifida. A bibliographical research regarding folate and NTD prevention, between 1988 and 2012, was done. The words used for Literature research were: Folic Acid, Spina bifida, Neural Tube Defects, Malformations. Search engines employed were: Medline, Pubmed, PopLine and the most relevant reports on the topic.
\end{abstract}

Keywords: Folic Acid, Spina bifida, Neural Tube Defects, Malformations

\section{Introduction}

Neural tube defects (NTDs) are congenital malformations of the brain and spinal cord caused by failure of the neural tube closure happening between 21 and 28 days following conception. ${ }^{[1-3]}$.

Among NTDs the most relevant are anencephaly, encephalocoeles and spina bifida. Anencephaly is caused by a failure of closure of the cranial neural arch and it's characterized by absence of variable amounts of brain, spinal cord nerve roots and meninges: usually the consequences are either stillbirth or neonatal death. Encephalocoeles is due to a closure failure of the anterior neural arch at a later stage of embryogenesis than anencephaly: sack containing brain tissue herniates through midline and usually it is associated with an occipital skull defect; with surgical therapy longterm outcome varies from normal function to severe multi- domain impairment

Furthermore, spina bifida is a consequence of a closure failure of the posterior vertebral arch; most commonly it affects the lumbo-sacral region and usually is related with hydrocephalus (blockage of drainage of the cerebrospinal fluid). Various kinds of spina bifida are known: herniation of the meninges through a defect in the lower spine (meningocoele) and other severe forms which include herniation of dysplastic spinal cord (myelomeningocoele). Hydrocephalus is a result of extra fluid spread around the brain causing an increase of intracranial pressure. Spina bifida includes variable levels of disability such as neurological (sensory and motor defects, learning disabilities, epilepsy), orthopaedic (contractures, joint dislocation, talipes) and functional (bladder and bowel dysfunctions). ${ }^{[4-5]}$ (Figure 1). 


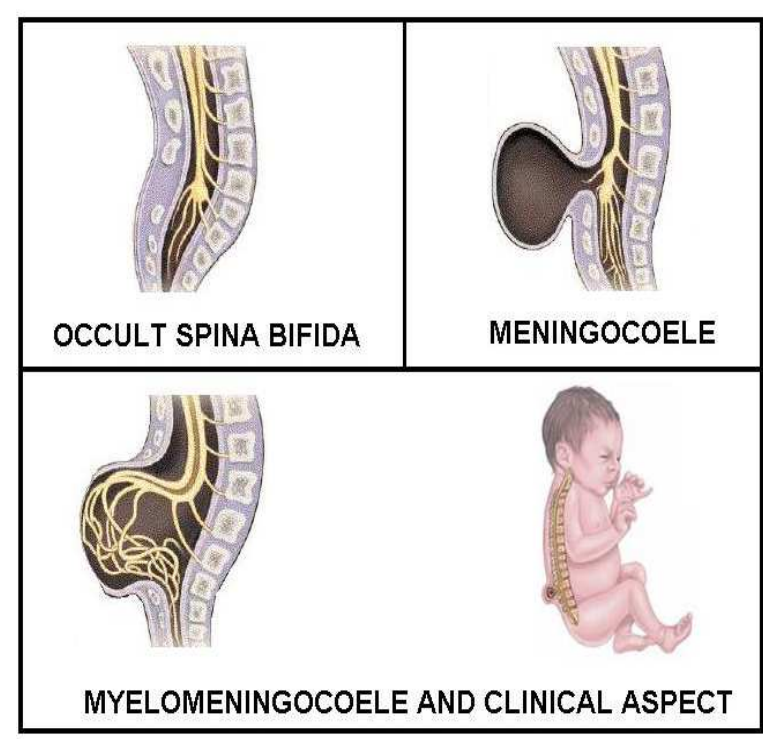

Fig. 1. Neural tube defects.

Almost 400.000 infants are born with NTDs each year all over the world. Although many advances have been made in the spina bifida treatment leading to an improved life quality, no treatment exists that will completely eliminate both serious disabilities and premature mortality. For these reasons, the main efforts of the physicians aim to prevent the risk of NTDs.

Besides, annual direct medical cost per patient was estimated to be about $€ 42.000$ for NTDs. ${ }^{[6]}$

It is widely accepted that adequate maternal consumption of folic acid before and during the early weeks of gestation can reduce the risk of having a child with a neural tube defect (NTD). As a result, public health authorities worldwide have recommended consuming $400 \mu \mathrm{g}$ folic acid per day during the periconceptional period. ${ }^{[7-10]}$ This recommended dose is based mainly on the amount of folic acid capable to reduce NTDs in the majority of epidemiological Literature studies. ${ }^{[11-15]}$

\section{Materials and Methods}

A bibliographical research regarding folate and NTD prevention, between 1988 and 2012, was done. The words used for Literature research were: Folic Acid, Spina bifida, Neural Tube Defects, Malformations. Search engines employed were the following: Medline, Pubmed, PopLine and the most relevant reports on the topic.

\section{Results}

The origin of NTDs can be explained by the interaction of genes and environmental factors (such as dietary deficiency). Several genetic and environmental factors contribute to their origin: the most established one is hyperhomocysteinemia. ${ }^{[16]}$ Folate and B12 are important determinants of plasma homocysteine and raised total homocysteine levels in maternal blood and amniotic fluid are associated with an increased risk of NTD pregnancies. ${ }^{[17]}$ Humans cannot produce folate. The most important source of folates are fresh and green leafy vegetables, citrus fruits and legumes.

Folic acid can be absorbed directly, while folate is changed into the monoglutamate form by conjugase enzymes; then, these monoglutamates are converted to dihydrofolate and to tetrahydrofolate (THF) by reducatase enzymes. THF is the precursor of all biologically active forms of this vitamin. When proteins are digested, aminoacids such as methionine are released, and methionine is converted to homocysteine. The last one is a toxic metabolite: for this reason, humans neutralize it as soon as possible. On the one hand, homocysteine is metabolized via the transsulfuration pathway to form cystathionine catalyzed by cystathionine -synthase and serine hydroxymethyltransferase. Cystathionine-synthase requires pyridoxal 5 -phosphate, i.e. vitamin B6, as a cofactor. On the other hand, remethylation of homocysteine to methionine is catalyzed by methionine synthase. This enzyme requires vitamin B 12 as a cofactor and 5-MTHF as the methyl donor. The latter explains the importance of folate-folic acid deficiency in the origin of NTD. The lower activity of the MethyleneTetraHydroFolateReductase (MTHFR) enzyme reduces the production of 5-MTHF and increases the plasma homocysteine level which causes a delay in the closure of the neural tube and thus indirectly NTD. If the mother is a homozygote for this mutation, the risk of NTD is 2-fold; if both the mother and fetus are homozygotes, the risk of NTD increases 6- to 7fold. Heterozygotes have a slight increase in the risk of NTD. A diet rich in folates is important for the prevention of NTD but cannot alone completely neutralize the genetic predisposition. $^{[16]}$

Periconceptional use of folic acid or multivitamins would be a simple and useful approach but the main problem is that about $50 \%$ of European pregnancies are unplanned. If women have unplanned pregnancies and are not using a supplement routinely, they cannot take advantage of this new preventive method during the preconceptional period. The explanation is clear: at the time of the first missed menstrual period and on about the 15 th postconceptional day, when the possible pregnancy is recognized, the neural tube is preparing to close. ${ }^{[18]}$ (Figure 2). 


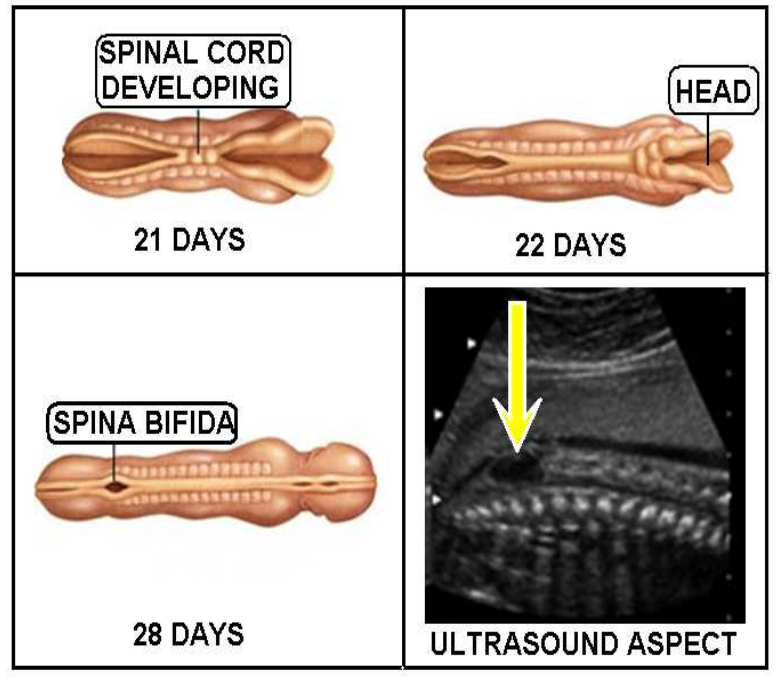

Fig. 2. SPINAL CORD DEVELOPMENT.

All women of childbearing age who are capable of becoming pregnant should consume $0.4 \mathrm{mg}$ of folic acid per day for the purpose of reducing their risk of having a pregnancy affected by spina bifida or other NTD' ${ }^{[19]}$

The supplementation with folic acid containing multivitamins is effective for the reduction of some others CAs. Hyperhomocysteinemia plays a role in the origin of NTD, and vitamins B 12, B 2, and B 6 are important cofactors in the folate-homocysteine metabolism.

Obviously, folate/folic acid is a key factor in homocysteine detoxication, but vitamins B 12, B 2, and B 6 also have a role in this biological mechanism and it may explain a higher efficacy of a multivitamin including these 4 'fetal protective' $\mathrm{B}$ vitamins as folic acid alone in the reduction of NDT.

In conclusion, folic acid-containing multivitamins seem to be more effective in the prevention of NTD, though obviously the use of folic acid alone is more simple and cheaper. ${ }^{[16]}$

At present, the use of $6 \mathrm{~S}-5-\mathrm{MTHF}$ seems to be better than the use of folic acid: the polymorphism of MTHFR mentioned before (:c.677C $>\mathrm{T}$ ) in folate metabolism is associated with an increased risk of NTD, but, in contrast to folic acid, the plasma response of 6S-5-MTHF is not affected in women carrying the common MTHFR:c.677C $>$ T variant. ${ }^{[20]}$

The previous major concern regarding the use of folic acid, mainly in higher doses, was its possible masking effect in patients with pernicious anemia. A low concentration of B 12 may compromise the activity of methionine synthase (which can reconvert homocysteine back to methionine as a form of 'detoxication'); thus cells suffer a pseudo-folate deficiency that suppresses DNA biosynthesis, which results in the clinical signs and symptoms of megaloblastic anemia. Folic acid bypasses this 'trap' because it can be reduced directly to THF, which can then be cycled to the folate forms used in DNA biosynthesis.

This treats the anemia but still allows plasma homocyste- ine concentrations to rise and continue the interruption to methylation reactions with the possible secondary outcome of demyelination of nerves, resulting in neuropathy. By contrast, supplementation with 6S-5- MTHF is not 'trapped', i.e. not recycled to THF, and therefore cannot 'mask' the clinical signs and symptoms of anemia and thus B 12 deficiency is more likely to be diagnosed and treated. [21]

6S-5-MTHF may be associated with a reduced interaction with antifolate drugs that inhibit dihydrofolate Reductase. ${ }^{[20]}$

6S-5-MTHF and folic acid showed comparable physiological activity, bioavailability, and absorption. ${ }^{[23]}$

Periconceptional folic acid or folic acid-containing multivitamin supplementation requires planned conception, and the proportion of females who prepare for conception is between only 30 and $75 \%$ in different countries.

The combination of oral contraceptive pills with folate is another option. ${ }^{[24]}$

Recently, the US FDA approved a new medicinal product comprised of drospirenone and ethinyl estradiol as contraceptive components and levomefolate calcium as a folate component.

Besides, it's well known that that the compliance of pill use is not perfect: infact, only $42 \%$ of oral contraceptive users remember to take daily their pill. ${ }^{[25]}$

Thus inadvertent 'accidental' conception may occur in about $2 \%$ of women who use contraceptive pills; the parallel use of folate is important for the prevention of NTD in their fetuses. ${ }^{[16]}$

Since the early 1990s there has been great progress in the efficacy of ultrasound diagnosis in the detection of NTD in fetuses. However, overall only $10-40 \%$ of all spina bifida cases are picked up during routine screening or discovered when other pathologies are detected. ${ }^{[22,26]}$ Nevertheless, all anencephalic fetuses are diagnosed by ultrasound in the second trimester, and about $80 \%$ of fetuses affected with spina bifida are also diagnosed on the basis of the characteristic 'lemon' and 'banana' signs which are specific changes in the head of affected fetuses. Mainly the diagnosis of fetuses with closed spina bifida is missed. After the prenatal diagnosis of NTD, informed pregnant women have two choices: continuing their pregnancy with the long-term medical, psychological, and financial consequences of their multiply handicapped children or deciding to terminate their pregnancy as soon as possible; the majority of women chooses the latter. The overall frequency of termination of pregnancy following prenatal diagnosis is $83 \%$ for anencephaly (range, $59-100 \%$ ) and $63 \%$ for spina bifida (range, $31-97 \%$ ). There are insufficient data to stratify the results for anencephaly; termination of pregnancy for spina bifida was more common when the prenatal diagnosis occurred at less than 24 weeks' gestation, with defects of greater severity. ${ }^{[27]}$

\section{Conclusions}


We can assert that folic acid can prevent about $70 \%$ of NTD conceptions while folic acid-containing multivitamin can prevent about $90 \%$. The diagnostic efficacy of ultrasound is nearly $100 \%$ in anencephalic fetuses and about $80 \%$ in fetuses with different manifestations of spina bifida. Elective termination of pregnancy due to NTD fetuses between the 16th and 20th gestation weeks induces serious psychological crises in many pregnant women. The risks of folic acid/multivitamin use are minimal, while the early complications and late adverse consequences of pregnancy terminations cannot be neglected. The cost of multivitamins and particularly folic acid is much lower than the onset of NTDs. As in many other cases, this confirms that prevention is better than cure (prevenire melius quam curare).

\section{Acknowledgments}

Valentina Pafumi has carried out English language editing for this article.

\section{Reference}

[1] Blencowe H., Cousens S., Modell B., Lawn J. "Folic acid to reduce neonatal mortality from neural tube disorders" - International Journal of Epidemiology 2010; 39: i110-i121.

[2] S.Sciarretta, V.Leanza FUNDAMENTAL OBSTETRIC AND GYNECOLOGIC ELEMENTS (Test Book) Edizione S.p.e. Catania.ISBN 978-88-96808-05-4 2011

[3] V. Leanza OSTETRICIA- EDIZIONI MINERVA MEDICA EDITOR S.P.A. 2009. (Test Book) ( ISBN 10: 88-7711-6315) ( ISBN 13: 978-88-7711-631-4).

[4] McIntosh N, Helms PJ, Smyth RL (eds). Forfar \& Arneil's. Textbook of Pediatrics. 6th edn. Churchill Livingstone: Edinburgh, 2003, pp. 902-907.

[5] Wyszynski DF (ed). Neural Tube Defects: from Origin to Treatment. US: Oxford University Press, 2006.

[6] Yi Y., Lindemann M., Colligs A., Snowball C. "Economic burden of neural tube defects and impact of prevention with folic acid: a literature review" - Eur J Pediatr (2011) 170: $1391-1400$.

[7] Department of Health Scottish Office Home and Health Department, Welsh Office, Department of Health and Social Services, Northern Ireland. Report from an Expert Advisory Group on Folic Acid and the Prevention of Neural Tube Defects; Department of Health: London, UK, 1992.

[8] Public Health Service, Centers for Disease Control and Prevention. Recommendations for the use of folic acid to reduce the number of cases of spina bifida and other neural tube defects. MMWR Morb. Mortal. Wkly. Rep. 1992, 41, $1-7$.

[9] National Health and Medical Research Council. Revised Statement on the Relationship between Dietary Folic Acid and Neural Tube Defects Such As Spina Bifida; NHMRC: Melbourne, Australia, 1993.

[10] Commission of the European Communities. Nutrient and
Energy Intakes for the European Community; Reports of the Scientific Committee for Food Thirty-First Series; Office for Official Publications of the European Communities: Luxembourg, 1993.

[11] Mulinare, J.; Cordero, J.F.; Erickson, J.D.; Berry, R.J. Periconceptional use of multivitamins and the occurrence of neural tube defects. JAMA 1988, 260, 3141-3145.

[12] Milunsky, A.; Jick, H.; Jick, S.S.; Bruell, C.L.; MacLaughlin, D.S.; Rothman, K.J.; Willett, W. Multivitamin/folic acid supplementation in early pregnancy reduces the prevalence of neural tube defects. JAMA 1989, 262, 2847-2852.

[13] Werler, M.M.; Shapiro, S.; Mitchell, A.A. Periconceptional folic acid exposure and risk of occurrent neural tube defects. JAMA 1993, 269, 1257-1261.

[14] Shaw, G.M.; Schaffer, D.; Velie, E.M.; Morland, K.; Harris, J.A. Periconceptional vitamin use, dietary folate, and the occurrence of neural tube defects. Epidemiology 1995, 6, 219-226.

[15] Khoury, M.J.; Shaw, G.M.; Moore, C.A.; Lammer, E.J.; Mulinare, J. Does periconceptional multivitamin use reduce the risk of neural tube defects associated with other birth defects? Data from two population-based case-control studies. Am. J. Med. Genet. 1996, 61, 30-36.

[16] Czeizel A., Dudàs I, paput L., Banhidy F. "Prevention of Neural-Tube defects with periconceptional folic acid, methylfolate, or multivitamins?" - Ann Nutr Metab 2011; 58: 263-271.

[17] Mills JL, McPartlin JM, Kirke PN, et al. Homocysteine metabolism in pregnancies complicated by neural-tube defects. Lancet. 1995; 345: 149-151.

[18] Busby A, Abramsky L, Dolk H et al: Preventing neural tube defects in Europe: a missed opportunity. Reprod Toxicol 2005; 20: 393-402

[19] CDC: Recommendations for the use of folic acid to reduce the number of cases of spina bifida and other neural tube defects. MMWR 1992; 41: 1233-1238.

[20] Prinz-Langenohl R, Brämswig S, Tobolski O, Smulders YM, Smith DE, Finglas PM, Pietrzik K: [6S]-5methyltetrahydrofolate increases plasma folate more effectively than folic acid in women with homozygous or wildtype $677 \mathrm{C}-\mathrm{T}$ polymorphism of methylenetetrahydrofolate reductase. Br J Pharmacol 2009; 158: 2014-2021.

[21] Wright AJ, King MJ, Finglas PM: Folate-supplemented oral contraceptives: does 6S-5- methytetrahydrofolic acid (Metafolin) offer advantages over folic acid? Gynaecol Forum 2010; $15: 29-32$.

[22] Pietrzik K, Bailey L, Shane B: Folic acid and L-5methyltetrahydrofolate: comparison of clinical pharmacokinetics and pharmacodynamics. Clin Pharmacokinet 2010; 49: $535-548$.

[23] Pietrzik K, Lamers Y, Brämswig S, Prinz- Langenohl R: Calculation of red blood cell folate steady state conditions and elimination kinetics after daily supplementation with various folate forms and doses in women in childbearing age. Am J Clin Nutr 2007; 86: 1414-1419.

[24] Holzgreve W: Adding folate to the pill to prevent neural tube defects. Gynaecol Forum 2010; 15: 4-8. 
[25] Oakley D, Sereika S, Bogue EL: Oral contraceptive pill use after an initial visit to a family planning clinic. Fam Plann Perspect 1991; 23: 151-154.

[26] Carbonaro A, Leanza V, Iozza I, Stracquadanio M, Formuso C, Ciotta L: screening for preeclampsia and iugr by doppler echo-flowmetry of the uterine district in the first trimester of pregnancy. International Journal of Gynecology and Obste- trics Volume 119, Supplement 3 (2012), Page S739.

[27] Johnson CY, Honein MA, Dana Flanders W, Howards PP, Oakley GP Jr, Rasmussen SA.Pregnancy termination following prenatal diagnosis of anencephaly or spina bifida: A systematic review of the literature. Birth Defects Res A Clin Mol Teratol. 2012 Nov; 94(11): 857-63. doi: 10.1002/ bdra.23086. Epub 2012 Oct 25. 